\title{
EL GRUPO DE HEISENBERG TRIDIMENSIONAL $\mathcal{H}_{3}$
}

\author{
Richard S. Quispe Rivas ${ }^{1}$
}

RESUMEN.- En vista de lo desarrollado en ([7]), tenemos la teoría necesaria para estudiar la geometría de los grupos de Heisenberg. El objetivo de este trabajo es llevar todos los resultados al caso tridimensional. Para esta empresa nos apoyamos en una de las bondades del teorema de Ado (ver [3]): "Toda álgebra de Lie de dimensión $n$ puede ser caracterizada dentro del álgebra de matrices cuadradas de orden $n \times n^{\prime \prime}$.

PALABRAS CLAVE.- Algebra de Lie, grupo de Heisemberg, generalizados, álgebra de Heisemberg.

\section{THE HEISENBERG'S TRIDIMENSIONAL GROUP $\mathcal{H}_{3}$}

ABSTRAC.- On the basis of [7], we have the necessary theory to study the Heisenberg's group geometry. This paper goal is to carry the whole results to the tridimensional case. We are helped by the characteristics of the Ado theorema (see [3]): "All Lie algebra of dimension $n$ can be characterized within the square matrix algebra of nxn".

KEYWORDS.- Lie algebra, Heisenberg's generalized group, Heisenberg's algebra.

\section{INTRODUCCIÓN}

Los grupos nilpotentes con métricas invariantes a izquierda juegan un rol importante en geometría riemanniana, en varias ocasiones aparecen en forma natural, por ejemplo en la descomposición de Iwasawa del grupo de isometrías de un espacio simétrico riemanniano no-compacto. Entre los grupos de Lie nilpotentes son de particular interés los de segundo orden.

Los grupos de Heisenberg generalizados forman una subclase de los grupos de Lie nilpotentes de orden dos simplemente conexos con métrica invariante a izquierda, ellos fueron introducidos por A. Kaplan (ver [6]) en 1980 al estudiar las ecuaciones diferenciales parciales hypoelípticas. Kaplan consideró una composición de formas cuadráticas, definiendo una clase de grupos de Lie nilpotentes de orden dos simplemente conexos con métrica invariante a izquierda, que incluyen los grupos de Heisenberg clásicos. Pero se sabe que la composición de formas cuadráticas está íntimamente relacionada a la teoría de los módulos Clifford. Efectivamente, a cada representación del álgebra de Clifford de $\mathrm{R}^{m}$ con respecto a una forma cuadrática definida negativa se le puede asociar un grupo de Heisenberg generalizado con centro de dimensión $m$.

${ }^{1}$ Magister en Matemáticas - PUCP - (Profesor UNMSM). 
Esta correspondencia es uno a uno, solamente si $m \neq z 3(\bmod 4)$, y ello nos permite obtener una clasificación completa de los grupos de Heisenberg generalizados usando la teoría de representación de las álgebras de Clifford (ver [1], [5], [9]).

Los resultados que se obtienen del análisis geométrico de los grupos de Heisenberg de dimensión 3 se pueden generalizar a grupos de Heisenberg que tienen dimensión $2 n+1$.

\section{PRELIMINARES}

Sean $U$ y $V$ dos espacios vectoriales reales de dimensiones $m$ y $n$ respectivamente. Consideremos en $\mathbf{n}:=U \times V$ un producto interno $\langle$,$\rangle , de tal manera que U$ sea ortogonal a $V$, y definamos una representación de $U$ en $V$ :

$$
J: U \longrightarrow \operatorname{End}(V)
$$

que cumpla

$$
|J(a) x|=|a||x|
$$

y

$$
J(a)^{2}=\langle a, a\rangle i d v,
$$

para $i d_{V}$ la aplicación identidad sobre $V ; x, y \in V ; a \in U$.

Observemos que al polarizar (2) y (3) obtenemos:

$$
\begin{aligned}
& \langle J(a) x, J(b) x\rangle=\langle a, b\rangle|x|^{2}, \\
& \langle J(a) x, J(a) y\rangle=|x|^{2}\langle x, y\rangle .
\end{aligned}
$$

De las igualdades (3) y (5) resulta

$$
\langle J(a) x, y\rangle+\langle x, J(a) y\rangle=0,
$$

y esto nos permite definir la aplicación bilineal $\beta: V \times V \rightarrow U$ del siguiente modo

$$
\langle a, \beta(x, y)\rangle=\langle J(a) x, y\rangle .
$$

Donde $x, y \in V ; a \in U$; apelando a (6) vemos que $\beta$ es antisimétrica.

Extendamos $\beta$ a $\mathbf{n}$ de la siguiente manera:

$$
[a+x, b+y]=\beta(x, y) \in U
$$

donde $x, y \in V, a, b \in U$, de tal forma que el conjunto

$$
\mathbf{n}=U \times V \approx U \oplus V
$$


adquiere una estructura de álgebra de Lie. Se puede ver fácilmente que $\mathbf{n}$ es nilpotente de orden dos con centro $U$, excepto en el caso abeliano, cuando $U=\{0\}$. Estas álgebras reciben el nombre de álgebras de Lie tipo $\mathbf{H}$ o álgebras de Heisenberg generalizados y a sus correspondientes grupos de Lie se les conoce como grupos de Heisenberg generalizados. Cuando $m=1$, $\mathbf{n}$ es un álgebra de Heisenberg y su grupo de Lie es llamado grupo de Heisenberg.

\section{EL ALGEBRA DE HEISENBERG TRIDIMENSIONAL $\mathfrak{h}_{3}$}

Consideremos $\operatorname{dim} \mathfrak{n}=3$ y denotemos esta álgebra de Lie por $\mathfrak{h}_{3}$, vale decir $\mathfrak{h}_{3}=U \oplus V$. Sean $\left\{e_{3}\right\}$ y $\left\{e_{1}, e_{2}\right\}$ bases ortogonales de $U$ y $V$, respectivamente y la aplicación $\beta: V \times V \rightarrow U$ que satisface

$$
\left\langle J\left(e_{3}\right) e_{1}, e_{2}\right\rangle=\left\langle e_{3}, \beta\left(e_{1}, e_{2}\right)\right\rangle \text {. }
$$

donde $J\left(e_{3}\right) \in \operatorname{End}(V)$. Considerando $\beta\left(e_{1}, e_{2}\right)=\left[e_{1}, e_{2}\right] \in U$, tenemos que $\left[e_{1}, e_{2}\right]=\lambda e_{3}$ para algún $\lambda \in \mathbb{R} ; \mathrm{y}$ así

$$
\left\langle J\left(e_{3}\right) e_{1}, e_{2}\right\rangle=\left\langle e_{3}, \lambda e_{3}\right\rangle=\lambda\left|e_{3}\right|=\lambda
$$

Pero, la propiedad que caracteriza a las álgebras de Heisenberg (ver [7]) establece que la aplicación

$$
\phi_{e_{i}}: V \rightarrow U
$$

dada por $\phi_{e_{i}}\left(e_{j}\right)=\left[e_{i}, e_{j}\right]$, es una isometría desde el complemento ortogonal del núcleo. de $\phi_{e_{i}}$ en $V$ sobre $U$ y dado que

$$
\left|e_{j}\right|=\left|\phi_{e_{i}}\left(e_{j}\right)\right|=\left|\left[e_{i}, e_{j}\right]\right|=1
$$

y $\left|\left[e_{i}, e_{j}\right]\right|=\left|\lambda e_{3}\right|$, entonces $|\lambda|=1$, para $i \neq j$.

En resumen se satisface

$$
\left\{\begin{array}{l}
{\left[e_{1}, e_{2}\right]=e_{3}} \\
{\left[e_{1}, e_{3}\right]=\left[e_{2}, e_{3}\right]=0 .}
\end{array}\right.
$$

Por otro lado, podemos determinar la matriz que representa $J\left(e_{3}\right)$ en la base $\left\{e_{1}, e_{2}\right\}$ de la siguiente manera: 


$$
\begin{aligned}
& \left\langle J\left(e_{3}\right) e_{1}, e_{2}\right\rangle=\left\langle e_{3},\left[e_{1}, e_{2}\right]\right\rangle=1 \\
& \left\langle J\left(e_{3}\right) e_{2}, e_{1}\right\rangle=\left\langle e_{3},\left[e_{2}, e_{1}\right]\right\rangle=1 ;
\end{aligned}
$$

haciendo $\alpha_{i j}=\left\langle J\left(e_{3}\right) e_{i}, e_{j}\right\rangle$, obtenemos

$$
\left(\alpha_{i j}\right)=\left(\begin{array}{rr}
0 & 1 \\
-1 & 0
\end{array}\right)
$$

Así para

$$
x=x_{1} e_{1}+x_{2} e_{2} \in V
$$

$\mathrm{y}$, por lo anteriormente establecido, se obtiene la regla de correspondencia para $J\left(e_{3}\right)$ via

$$
J\left(e_{3}\right) x=\left(\begin{array}{rr}
0 & 1 \\
-1 & 0
\end{array}\right)\left(\begin{array}{l}
x_{1} \\
x_{2}
\end{array}\right)=\left(x_{2}, x_{1}\right) .
$$

Luego de conocer el comportamiento de la aplicación $J\left(e_{3}\right)$, en las siguientes secciones veremos, que la geometría del grupo de Heisenberg tridimensional podrá ser caracterizada por el valor $J\left(e_{3}\right)$.

Denotemos por $\mathrm{H}_{3}$ el grupo de Lie simplemente correspondiente al álgebra de Lie $\mathfrak{h}_{3}$, de esta manera, la aplicación exponencial resulta ser un difeomorfismo global (ver [4]), y en particular, el grupo de Heisenberg tridimensional será determinado globalmente por su álgebra $\mathfrak{h}_{3}$ vía la aplicación exponencial; todo ello será detallado a continuación.

\section{APLICACIÓN EXPONENCIAL EN $\mathcal{H}_{3}$}

Por el teorema de Ado, el álgebra de Heisenberg tridimensional es isomorfa al álgebra de las matrices cuadradas $3 \times 3$ triangulares superiores con ceros en la diagonal, donde la operación corchete $[$, ] está dado por

$$
[A, B]=A B-B A,
$$

donde $A B$ indica el producto de las matrices $A$ y $B$. Es fácil ver que esta álgebra es nilpotente de orden 2. Asímismo, el correspondiente grupo de Lie nilpotente simplemente conexo será denotado por $\mathcal{H}_{3}$, donde la operación de grupo es la multiplicación usual de matrices. Entonces podemos considerar a la aplicación

$$
\exp : \mathfrak{h}_{3} \rightarrow \mathcal{H}_{3}
$$


como exponencial para matrices; vale decir, $\operatorname{si} \exp (A)=e^{A}$ se tiene

$$
e^{A}=I+A+\frac{1}{2 !} A^{2}+\ldots+\frac{1}{j !} A^{3}+\ldots
$$

Sea $A \in \mathfrak{h}_{3}$, la matriz $A$ tendrá la forma

$$
A=\left(\begin{array}{lll}
0 & a & c \\
0 & 0 & b \\
0 & 0 & 0
\end{array}\right),
$$

donde $a, b, c \in \mathbb{R}$; lo cual lleva inevitablemente a

$$
\begin{aligned}
e^{A} & =\left(\begin{array}{lll}
1 & 0 & 0 \\
0 & 1 & 0 \\
0 & 0 & 1
\end{array}\right)+\left(\begin{array}{lll}
0 & a & c \\
0 & 0 & b \\
0 & 0 & 0
\end{array}\right)+\frac{1}{2}\left(\begin{array}{ccc}
0 & 0 & a b \\
0 & 0 & 0 \\
0 & 0 & 0
\end{array}\right) \\
& =\left(\begin{array}{ccc}
1 & a & c+\frac{a b}{2} \\
0 & 1 & b \\
0 & 0 & 1
\end{array}\right) .
\end{aligned}
$$

Se puede ver fácilmente que las matrices que forman el centro de $\mathfrak{h}_{3}$ son de la forma

$$
\left(\begin{array}{lll}
0 & 0 & c \\
0 & 0 & 0 \\
0 & 0 & 0
\end{array}\right)
$$

Mediante el isomorfismo de espacios vectoriales

$$
\left(\begin{array}{lll}
0 & a & c \\
0 & 0 & b \\
0 & 0 & 0
\end{array}\right) \mapsto(a, b, c)
$$

podemos incluir la estructura de álgebra de $\mathfrak{h}_{3}$ en $\mathbb{R}^{3}$, la cual denotaremos por $\left(\mathbb{R}^{3},[],\right)$. Entonces, la aplicación

$$
\exp :\left(\mathbb{R}^{3},[,]\right) \rightarrow \mathcal{H}_{3}
$$

está dada de la siguiente manera:

$$
\exp ((a, b, c))=\left(\begin{array}{ccc}
1 & a & c+\frac{a b}{2} \\
0 & 1 & b \\
0 & 0 & 1
\end{array}\right)
$$


Como la aplicación exponencial es un difeomorfismo global, induciremos la operación

* en $\mathbb{R}^{3}$, de tal manera que exp sea un isomorfismo de grupos. Es decir

$$
\left.\left.\exp \left(\left(a, b_{1}, c_{1}\right) *\left(a_{2}, b_{2}, c_{2}\right)\right)=\exp \left(a_{1}, b_{1}, c_{1}\right)\right) \exp \left(a_{2}, b_{2}, c_{2}\right)\right)
$$

Haciendo

$$
\exp \left((x, y, z)=\left(a_{1}, b_{1}, c_{1}\right) *\left(a_{2}, b_{2}, c_{2}\right)\right.
$$

tenemos

$$
\exp ((x, y, z))=\left(\begin{array}{ccc}
1 & a_{1} & c_{1}+\frac{a_{1} b_{1}}{2} \\
0 & 1 & b_{1} \\
0 & 0 & 1
\end{array}\right)\left(\begin{array}{ccc}
1 & a_{2} & c_{2}+\frac{a_{2} b_{2}}{2} \\
0 & 1 & b_{2} \\
0 & 0 & 1
\end{array}\right),
$$

luego

$$
\left(\begin{array}{ccc}
1 & x & z+\frac{x y}{2} \\
0 & 1 & y \\
0 & 0 & 1
\end{array}\right)=\left(\begin{array}{ccc}
1 & a_{1}+a_{2} & c_{1}+c_{2}+a_{1} b_{2}+\frac{a_{1} b_{1}}{2}+\frac{a_{2} b_{2}}{2} \\
0 & 1 & b_{1}+b_{2} \\
0 & 0 & 1
\end{array}\right) .
$$

Igualando componente a componente se tiene

$$
\left\{\begin{array}{l}
x=a_{1}+b_{2} \\
y=b_{1}+b_{2} \\
z=c_{1}+c_{2}+\frac{1}{2}\left(a_{1} b_{2}-a_{2} b_{1}\right)
\end{array}\right.
$$

Ello significa que la operación inducida $*$ en $\mathbb{R}^{3}$ está dada por

$$
\left(a_{1}, b_{1}, c_{1}\right) *\left(a_{2}, b_{2}, c_{2}\right)=\left(a_{1}+a_{2}, b_{1}+b_{2}, c_{1}+c_{2}+\frac{1}{2}\left(a_{1} b_{2}-a_{2} b_{1}\right)\right.
$$

De éste modo, se tiene que $\mathcal{H}_{3}$ y $\left(\mathbb{R}^{3}, *\right)$ son grupos de Lie isomorfos y ésta es la representación que usaremos, es decir, veremos a $\mathcal{H}_{3}$ como $\mathbb{R}^{3}$ provista de la operación *.

La aplicación traslación izquierda

$$
L_{(x, y, z)}: \mathcal{H}_{3} \rightarrow \mathcal{H}_{3}
$$

está dada por

$$
\begin{aligned}
L_{(x, y, z)}(a, b, c) & =(x, y, z) *(a, b, c) \\
& =\left(a+x, b+y, c+z+\frac{1}{2}(x b-y a)\right),
\end{aligned}
$$


y el diferencial en el punto $p=(a, b, c)$ es

$$
d L_{p}=\left(\begin{array}{rrr}
1 & 2 & \frac{b}{2} \\
0 & 1 & -\frac{a}{2} \\
0 & 0 & 1
\end{array}\right) .
$$

Los campos invariantes a izquierda generados por $\left\{e_{1}, e_{2}, e_{3}\right\}$ están dados por

$$
\begin{aligned}
& E_{1}(p)=\left(d L_{p}\right)\left(e_{1}\right)=(1,0,0)=\frac{\partial}{\partial x} \\
& E_{2}(p)=\left(d L_{p}\right)\left(e_{2}\right)=(0,1,0)=\frac{\partial}{\partial y} \\
& E_{3}(p)=\left(d L_{p}\right)\left(e_{3}\right)=\left(\frac{b}{2},-\frac{a}{2}, 1\right)=\frac{b}{2} \frac{\partial}{\partial x}-\frac{a}{2} \frac{\partial}{\partial y}+\frac{\partial}{\partial z} .
\end{aligned}
$$

De esta manera, hemos expresado los elementos de la base $\left\{e_{1}, e_{2}, e_{3}\right\}$ en términos de los campos coordenados $\left\{\frac{\partial}{\partial x}, \frac{\partial}{\partial y}, \frac{\partial}{\partial z}\right\}$. Por lo tanto, la métrica invariante a izquierda se expresa como

$$
d s^{2}=d x^{2}+d y^{2}+\frac{1}{4}\left(a^{2}+b^{2}+4\right)+\frac{b}{2} d x d z-\frac{b}{2} d z d x-\frac{a}{2} d y d z-\frac{a}{2} d z d y
$$

Observamos que, se ha determinado en forma explícita la métrica invariante a izquierda con la que se comporta el grupo de Heisenberg $\mathcal{H}_{3}$ De esta manera, estamos en condiciones de estudiar las propiedades geométricas de $\mathcal{H}_{3}$.

\section{CONEXIÓN Y CURVATURA}

Veremos en primer lugar la conexión riemanniana. Bastará determinarla en los elementos de la base del álgebra $\mathfrak{h}_{3}$. Puesto que, cada $e_{i}$ de la base genera un campo invariante a izquierda $E_{i}$, no habrá confusión si denotaremos por $e_{i}$ a estos últimos. Como la conexión riemanniana de campos invariantes a izquierda es también invariante a izquierda, se tiene

$$
\nabla_{e 1} e_{2}=a_{12} e_{1}+b_{12} e_{2}+c_{12} e_{3}
$$

donde $a_{12}, b_{12}, c_{12} \in \mathbb{R}$. Considerando que la base $\left\{e_{1}, e_{2}, e_{3}\right\}$ es ortonormal, y además usando la identidad 


$$
\left\langle\nabla_{\mathrm{X}} Y, Z\right\rangle=\frac{1}{2}(\langle[X, Y], Z\rangle-\langle[Y, Z], X\rangle+\langle[Z, X], Y\rangle),
$$

se obtiene

$$
\left\{\begin{array}{l}
a_{12}=\left\langle\nabla_{e 1} e_{2}, e_{1}\right\rangle=0 \\
b_{12}=\left\langle\nabla_{e 1} e_{2}, e_{2}\right\rangle=0 \\
c_{12}=\left\langle\nabla_{e 1} e_{2}, e_{3}\right\rangle=\frac{1}{2},
\end{array}\right.
$$

y con ello

$$
\nabla_{e 1} e_{2}=\frac{1}{2} e_{3}
$$

Siguiendo el mismo procedimiento se tendrá

$$
\left\{\begin{array}{l}
\nabla_{e 1} e_{3}=\nabla_{e 3} e_{1}=-\frac{1}{2} e_{2} \\
\nabla_{e 2} e_{3}=\nabla_{e 3} e_{2}=\frac{1}{2} e_{1} \\
\nabla_{e 2} e_{1}=-\frac{1}{2} e_{3} .
\end{array}\right.
$$

Por otro lado, el tensor curvatura de Riemann que satisface:

$$
R(X, Y)=\nabla_{X} \nabla_{Y}-\nabla_{Y} \nabla_{X}-\nabla_{[X, Y]}
$$

está dado por

$$
\left\{\begin{array}{l}
R(x, y) z=\frac{1}{4}(2 J([x, y]) z-J([y, z]) x-J([z, x]) y) \\
R(x, a) y=-\frac{1}{4}[x, J(a) y] \\
R(x, a) b=-\frac{1}{4} J(a) J(b) x \\
R(a, b) c=0 .
\end{array}\right.
$$

Usando lo establecido en (8), podemos hallar la curvatura de Riemann; por ejemplo

$$
\left\{\begin{array}{l}
R\left(e_{1}, e_{1}\right) e_{3}=0 \\
R\left(e_{2}, e_{1}\right) e_{2}=\frac{3}{4} e_{1} \\
R\left(e_{1}, e_{3}\right) e_{1}=\frac{3}{4} e_{3} .
\end{array}\right.
$$


De esta manera, usando (8), se tiene que la función curvatura

$$
K(X, Y):=-\langle R(X, Y) X, Y\rangle
$$

viene presentada por:

$$
\left\{\begin{array}{l}
K(x, y)=-\frac{3}{4}\langle(J[x, y]) x, y\rangle=-\frac{3}{4}|[x, y]|^{2} \\
K(x, a)=+\frac{3}{4}\langle[J(a) x, y], a\rangle=+\frac{1}{4}|x|^{2}|a|^{2} \\
K(a, b)=0 .
\end{array}\right.
$$

Luego, al evaluar lo dicho en los elementos de la base de $\mathfrak{h}_{3}$, y considerando (9), la función curvatura estará dada por

$$
\left\{\begin{array}{l}
K\left(e_{1}, e_{2}\right)=-\frac{3}{4} \\
K\left(e_{1}, e_{3}\right)=\frac{1}{4} \\
K\left(e_{1}, e_{3}\right)=\frac{1}{4} .
\end{array}\right.
$$

Como $\left\{e_{1}, e_{2}, e_{3}\right\}$ es una base ortonormal, la función curvatura coincide con la curvatura seccional y tendremos un espacio de curvatura no constante.

\section{GEODÉSICAS}

Ahora calculemos las geodésicas del grupo de Heisenberg. Observemos que la elección de las bases $\left\{e_{1}, e_{2}\right\}$ y $\left\{e_{3}\right\}$ de $V$ y $U$ definen las coordenadas $\left\{x_{1}, x_{2}\right\}$ y $\left\{x_{3}\right\}$ respectivamente, y entonces, para cada $x \in V$ y $a \in U$ se tiene

$$
\begin{aligned}
& x=x_{1} e_{1}+x_{2} e_{2} \\
& a=x_{3} e_{3} .
\end{aligned}
$$

En ([7]) se establece que los campos coordenados se pueden expresar de la forma

$$
\left\{\begin{array}{l}
\frac{\partial}{\partial x_{1}}=e_{1}-\frac{1}{2} x_{2} e_{3} \\
\frac{\partial}{\partial x_{2}}=e_{2}+\frac{1}{2} x_{1} e_{3} \\
\frac{\partial}{\partial x_{3}}=e_{3} .
\end{array}\right.
$$


Luego, una curva $t \rightarrow \gamma(t) \in \mathcal{H}_{3}$ será descrita por medio de funciones vectoriales $t \rightarrow x(t) \in V$ y $t \rightarrow a(t) \in U$, y considerando además, que exp es un difeomorfismo global, podemos reescribir $\gamma(t)=\exp (x(t)+a(t))$, el cual tiene por vector tangente a

$$
\frac{d \gamma}{d t}=\dot{x}_{1} \frac{\partial}{\partial x_{1}}+\dot{x}_{2} \frac{\partial}{\partial x_{2}}+\dot{x}_{3} \frac{\partial}{\partial x_{3}} .
$$

Reemplazando (10) en (11), obtenemos

$$
\frac{d \gamma}{d t}=\dot{x}_{1} e_{1}+\dot{x}_{2} e_{2}+\frac{1}{2}\left(x_{1} \dot{x}_{2}-x_{1} \dot{x}_{2}+2 \dot{x}_{3}\right) e_{3} .
$$

Además, se sabe que $\gamma$ será una geodésica si y sólo si

$$
\nabla_{\frac{d \gamma}{d t}} \frac{d \gamma}{d t}=\frac{D}{d t}\left(\frac{d \gamma}{d t}\right)=0
$$

y así, la reemplazar (12) en (13) obtenemos

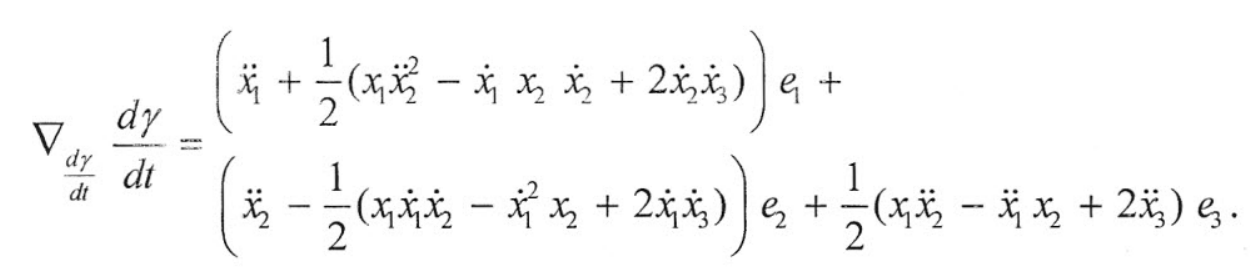

En consecuencia, $\nabla_{\frac{d \gamma}{d t}} \frac{d \gamma}{d t}=0$ si y sólo si

$$
\left\{\begin{array}{l}
x_{1}+\frac{x_{1} \dot{x}_{2}^{2}}{2}-\frac{\dot{x}_{1} x_{2} \dot{x}_{2}}{2}+\dot{x}_{2} \dot{x}_{3}=0 \\
x_{2}-\frac{x_{1} \dot{x}_{1} \dot{x}_{2}}{2}+\frac{\dot{x}_{1}^{2} x_{2}}{2}-\dot{x}_{1} \dot{x}_{3}=0 \\
x_{3}-\frac{x_{1} \dot{x}_{2}}{2}-\frac{x_{1} x_{2}}{2}=0
\end{array}\right.
$$

El sistema anterior se puede expresar como

$$
\left\{\begin{array}{l}
\ddot{x}_{1}+\dot{x}_{2}\left(\frac{x_{1} \dot{x}_{2}}{2}-\frac{\dot{x}_{1} x_{2}}{2}+\dot{x}_{3}\right)=0 \\
x_{2}-\dot{x}_{1}\left(\frac{x_{1} \dot{x}_{2}}{2}-\frac{\dot{x}_{1} x_{2}}{2}+\dot{x}_{3}\right)=0 \\
x_{3}+\left(\frac{x_{1} x_{2}}{2}-\frac{x_{1} x_{2}}{2}\right)=0
\end{array}\right.
$$


lo cual implica en particular

$$
\frac{d}{d t}\left(x_{1} \dot{x}_{2}-\dot{x}_{1} x_{2}\right)=x_{1} \ddot{x}_{2}-\ddot{x}_{1} x_{2},
$$

de donde, la tercera ecuación de (14) resulta

$$
\ddot{x}_{3}=\frac{d}{d t}\left(\frac{x_{1} \dot{x}_{2}}{2}-\frac{\dot{x}_{1} x_{2}}{2}\right),
$$

lo cual deriva en

$$
\dot{x}_{3}=-\frac{1}{2}\left(x_{1} \dot{x}_{2}-\dot{x}_{1} x_{2}\right)+C
$$

donde $C$ es una constante.

Nos limitaremos a estudiar geodésicas que pasan por el origen, es decir $x_{i}(0)=0$ para $i=1,2,3$. Se tiene los siguientes casos:

Cuando $\dot{x}_{1}(0) \neq 0, \dot{x}_{2}(0) \neq 0$ y $\dot{x}_{3}(0)=0$, recordando que las geodésicas pasan por el origen, de (15) resulta $C=0$, y de esta manera

$$
\dot{x}_{3}=-\frac{1}{2}\left(x_{1} \dot{x}_{2}-\dot{x}_{1} x_{2}\right),
$$

así, en la primera ecuación de (14) se lee

$$
\ddot{x}_{1}-\dot{x}_{2}\left(-\dot{x}_{3}+\dot{x}_{3}\right)=0 \text {, }
$$

con lo cual $x_{1}=0$, es decir, $x_{1}(t)=\dot{x}_{1}(0) t$. De la misma manera, se obtiene que $x_{2}(t)=\dot{x}_{2}(0) t$. Asímismo $\dot{x}_{3}=0$, lo cual implica $x_{3}=C_{3}$. Pero evaluando en $t=0$ resulta $C_{3}=0$ y en consecuencia $x_{3}(t)=0$. Se observa que la solución del sistema (14) es

$$
\left\{\begin{array}{l}
x_{1}(t)=\dot{x}_{1}(0) t \\
x_{2}(t)=\dot{x}_{2}(0) t \\
x_{3}(t)=0
\end{array}\right.
$$

es decir, son rectas contenidas en el plano $x_{1} x_{2}$.

Si $\dot{x}_{1}(0)=\dot{x}_{2}(0)=0 \quad y \quad \dot{x}_{3}(0) \neq 0$, hacemos $t=0$ en (15) y resulta $C=\dot{x}_{3}(0)$. Por lo tanto,

$$
\dot{x}_{3}=-\frac{1}{2}\left(x_{1} \dot{x}_{2}-\dot{x}_{1} x_{2}\right)+\dot{x}_{3}(0) \text {. }
$$


Luego, del sistema (14) obtenemos

$$
\left\{\begin{array}{l}
x_{1}+\dot{x}_{3}(0) \dot{x}_{2}=0 \\
x_{2}-\dot{x}_{3}(0) \dot{x}_{1}=0 .
\end{array}\right.
$$

Resolviendo el sistema (16) se deduce $x_{1}(t)=x_{2}(t)=0$. Con estos valores resulta $x_{3}(t)=\dot{x}_{3}(0) t$, y la solución de (14) será

$$
\left\{\begin{array}{l}
x_{1}(t)=0 \\
x_{2}(t)=0 \\
x_{3}(t)=\dot{x}_{3}(0) t
\end{array}\right.
$$

una recta vertical.

Si $\dot{x}_{1}(0) \neq 0, \dot{x}_{2}(0) \neq 0 \quad y \quad \dot{x}_{3}(0) \neq 0$, con estas condiciones, de (15) obtenemos

$$
\dot{x}_{3}(t)=-\frac{1}{2}\left(x_{1} \dot{x}_{2}-\dot{x}_{1} x_{2}\right)+\dot{x}_{3}(0),
$$

y reemplazando en las dos primeras igualdades de (14), se tiene

$$
\left\{\begin{array}{l}
\ddot{x}_{1}+\dot{x}_{3}(0) \dot{x}_{2}=0 \\
\ddot{x}_{2}+\dot{x}_{3}(0) \dot{x}_{1}=0 .
\end{array}\right.
$$

$\mathrm{Al}$ integrar y forzar las condiciones iniciales, obtenemos el siguiente sistema de primer orden

$$
\left\{\begin{array}{l}
\dot{x}_{1}+\dot{x}_{3}(0) x_{2}=\dot{x}_{1}(0) \\
\dot{x}_{2}+\dot{x}_{3}(0) x_{1}=\dot{x}_{2}(0)
\end{array}\right.
$$

cuya solución está dado por

$$
\left\{\begin{array}{l}
x_{1}=\frac{\dot{x}_{2}(0)}{\dot{x}_{3}(0)} \cos \left(\dot{x}_{3}(0) t\right)+\frac{\dot{x}_{1}(0)}{\dot{x}_{3}(0)} \sin \left(\dot{x}_{3}(0) t\right)-\frac{\dot{x}_{2}(0)}{\dot{x}_{3}(0)} \\
x_{2}=\frac{\dot{x}_{1}(0)}{\dot{x}_{3}(0)} \cos \left(\dot{x}_{3}(0) t\right)+\frac{\dot{x}_{2}(0)}{\dot{x}_{3}(0)} \sin \left(\dot{x}_{3}(0) t\right)+\frac{\dot{x}_{1}(0)}{\dot{x}_{3}(0)} .
\end{array}\right.
$$

Notemos que

$$
\begin{aligned}
\left(x_{1}+\frac{\dot{x}_{1}(0)}{\dot{x}_{3}(0)}\right)^{2}+\left(x_{2}+\frac{\dot{x}_{2}(0)}{\dot{x}_{3}(0)}\right)^{2} & =\left(\frac{\dot{x}_{1}(0)}{\dot{x}_{3}(0)}\right)^{2}+\left(\frac{\dot{x}_{2}(0)}{\dot{x}_{3}(0)}\right)^{2} \\
& =\frac{\dot{x}_{1}^{2}(0)+\dot{x}_{2}^{2}(0)}{\dot{x}_{3}^{2}}
\end{aligned}
$$


es decir, las geodésicas que pasan por el origen son espirales alrededor del cilindro de radio

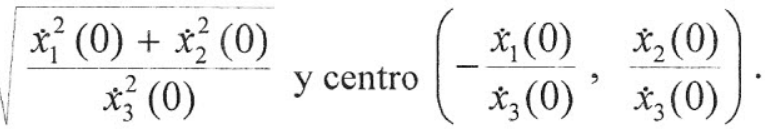

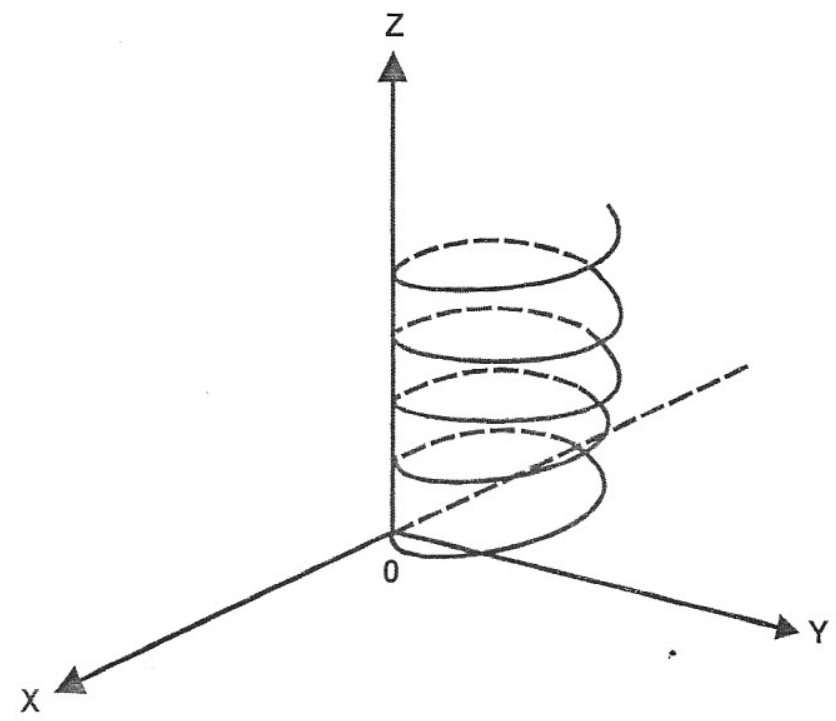

Figura 1: Geodésicas en el grupo de Heisenberg $\mathcal{H}_{3}$

\section{GRUPOS DE ISOMETRÍAS}

Consideremos ahora el grupo de isometrías de $\mathfrak{h}_{3}$ (ver [7]). Sea el automorfismo $\varphi: \mathcal{H}_{3} \rightarrow \mathcal{H}_{3}$ que preserva producto interno, tal que $\varphi(0)=0$. Sabemos que

$$
\operatorname{Isom}\left(\mathfrak{h}_{3}\right)=\mathcal{H}_{3} \times A\left(\mathcal{H}_{3}\right)
$$

donde $A\left(\mathcal{H}_{3}\right)$ son automorfismos que preservan producto interno. La diferencial de $\psi$ en la identidad.

$$
d \psi_{0}: T_{0}\left(\mathfrak{h}_{3}\right) \rightarrow T_{0}\left(\mathfrak{h}_{3}\right)
$$

tiene en la base $\left\{e_{1}, e_{2}, e_{3}\right\}$ representación matricial

$$
A=d \psi_{0}=\left(\begin{array}{lll}
a_{11} & a_{12} & a_{13} \\
a_{21} & a_{22} & a_{23} \\
a_{31} & a_{32} & a_{33}
\end{array}\right) .
$$

Además, $d \psi_{0}$ es un automorfismo de álgebras, es decir 


$$
\left[d \psi_{0}\left(e_{1}\right), d \psi_{0}\left(e_{2}\right)\right]=d \psi_{0}\left[e_{1}, e_{2}\right]=d \psi_{0}\left(e_{3}\right) .
$$

Como A es una isometría, se tiene

$$
A^{t} A=I
$$

pero sabemos que, $d \psi_{0}\left(e_{i}\right) \in \mathfrak{h}_{3}$ para $i=1,2,3$ y entonces

$$
d \psi_{0}\left(e_{i}\right)=\sum_{j=1}^{3} a_{i j} e_{j} .
$$

Reemplazando (19) en (17) tenemos

$$
\left[\sum_{j=1}^{3} a_{i j} e_{j}, \sum_{k=1}^{3} a_{k 2} e_{k}\right]=\left(a_{12} a_{22}-a_{21} a_{22}\right) e_{3}=a_{13} e_{1}+a_{23} e_{1}+a_{23} e_{2}+a_{33} e_{3},
$$

de donde

$$
\begin{aligned}
a_{12} a_{22}+a_{21} a_{22} & =a_{33} \\
a_{13} & =a_{23}=0 .
\end{aligned} .
$$

De (18) obtenemos

$$
\sum_{j=1}^{3} a_{j i}^{2}=1
$$

para $i=1,2,3$, además de

$$
\begin{aligned}
& a_{12} a_{11}+a_{22} a_{21}+a_{32} a_{31}=0 \\
& a_{31} a_{11}+a_{23} a_{21}+a_{33} a_{31}=0 \\
& a_{13} a_{12}+a_{23} a_{22}+a_{33} a_{32}=0 .
\end{aligned}
$$

Combinando los resultados anteriores se llega a

$$
\begin{aligned}
& a_{33}= \pm 1, \\
& a_{12}=a_{21}, \\
& a_{31}=a_{32}=0 ;
\end{aligned}
$$

y reemplazando lo que se obtuvo en la segunda igualdad de (20) en (21), se concluye

$$
\begin{aligned}
& a_{11}^{2}+a_{12}^{2}=1 \\
& a_{21}^{2}+a_{22}^{2}=1 .
\end{aligned}
$$

Haciendo,

$$
a_{i j}=\left\{\begin{array}{l}
\sin \varphi, \text { para } i=j \\
\cos \varphi, \text { para } i \neq j,
\end{array}\right.
$$


tendremos

$$
d \psi_{0}=\left(\begin{array}{ccc}
\sin \varphi & \cos \varphi & 0 \\
\cos \varphi & \sin \varphi & 0 \\
0 & 0 & 1
\end{array}\right)
$$

De esta manera, hemos hallado la diferencial de una aplicación aún por conocer, para lo cual consideremos la siguiente aplicación

$$
\omega(x, y, z)=(x \sin \varphi+\cos \varphi, x \cos \varphi+y \sin \varphi, z),
$$

es fácil verificar que $d \psi_{0}=d \omega_{0}$, además $\psi(0)=\omega(0)$. Luego, según [2] (pág, 163) o también [8] (pág. 62), podemos afirmar que $\psi=\omega$. Por lo tanto,

$$
\psi(x, y, z)=(x \sin \varphi+y \cos \varphi, x \cos \varphi+y \sin \varphi, z) .
$$

Se puede observar que todas ellas son rotaciones alrededor del eje $Z$.

\section{REFERENCIAS BIBLIOGRÁFICAS}

[1] Atiyah, M. F., Boot, R., Shapiro, A., Clifford Modules, Topology 3, Suppl. 1, 3-38, 1964.

[2] Carmo, M. Do, Geometria Riemanniana, IMPA, Rio de Janeiro, 1988.

[3] Cuadros, Jaime, Introducción Sistemática a Grupos y Álgebras de Lie, Tesis de Licenciatura, PUCP, Lima, 1991.

[4] Helgason, S., Diferential Geometry and Simmetric Spaces, Academic Press., 1948.

[5] J. Berndt, F. Tricerri, L. Vanhecke, Generalized Heisenberg Groups and DamekRicci Harmanic Spaces, Academic Press., Springer Verlag, Berlin, 1995.

[6] Kaplan, A., Fundamental solutions for a class of hypoellytic PDE generated by composition of quadratics forms, Trans. Amer. Math. Soc. 258, 147-153, 1980.

[7] Kaplan, A., Riemman Nilmanifolds Attached to Cliffford Modules, Geom. Dedicata $11,127-136,1981$.

[8] Kobayashi, S., Nomizu, K., Foundations of Diferential Geometry, Interscience Publishers, New York and London, 1963.

[9] Lam, T. Y., The Algebraic Theory of Quadratic Forms, Benjamin, 1973. 